\title{
Fatores de Risco para Problemas de Saúde Mental na Infância/Adolescência ${ }^{1}$
}

\author{
Daniel Graça Fatori de Sá \\ Universidade Presbiteriana Mackenzie \\ Isabel A. Santos Bordin \\ Universidade Federal de São Paulo \\ Denise Martin \\ Universidade Federal de São Paulo e Universidade Católica de Santos \\ Cristiane S. de Paula ${ }^{2}$ \\ Universidade Federal de São Paulo e Universidade Presbiteriana Mackenzie
}

\begin{abstract}
RESUMO - Estudo de corte transversal conduzido em comunidade urbana de baixa renda do município de Embu-SP com objetivo de identificar fatores associados a problemas de saúde mental em crianças/adolescentes (PSMCA) em amostra probabilística ( $\mathrm{N}=67$, faixa etária 4-17 anos). Foram aplicados instrumentos estruturados às mães: Child Behavior Checklist (PSMCA); WorldSAFE Core Questionnaire (dados sociodemográficos; violência doméstica; embriaguez do pai/padrasto); Self-Report Questionnaire (problemas de saúde mental maternos, ideação suicida materna). Os resultados do estudo apontaram fatores estatisticamente associados aos PSMCA: criança/adolescente ser do sexo masculino e sofrer punição física grave; ideação suicida da mãe e violência conjugal física grave contra a mãe; embriaguez do pai/padrasto. Concluindo, grupos vulneráveis com características individuais/familiares identificadas neste estudo devem ser considerados prioritários em propostas de prevenção/tratamento.
\end{abstract}

Palavras-chave: saúde mental; fatores de risco; criança; adolescente; estudos transversais.

\section{Risk Factors for Mental Health Problems in Childhood/Adolescence.}

\begin{abstract}
A cross-sectional study was conducted in an urban low-income community in the municipality of Embu-SP with the objective of identifying factors associated with mental health problems of children and adolescents (MHPCA) in a probabilistic sample ( $\mathrm{N}=67$, aged 4-17 years). Standardized instruments were applied to mothers: Child Behavior Checklist; WorldSAFE Core Questionnaire (demographics, domestic violence, father/stepfather drunkenness); Self-Report Questionnaire (maternal mental health problems and suicide ideation). The results of this study identified factors statistically related to MPHCA: child/adolescent of male gender and severe physical punishment; maternal suicide ideation and severe physical marital violence; father/stepfather drunkenness. As a result, vulnerable groups with individual/family characteristics identified in this study should have priority in prevention/treatment programs.
\end{abstract}

Keywords: mental health; risk factors; child; adolescent; cross-sectional studies.

Estudos epidemiológicos na área de saúde mental da infância e adolescência eram escassos nos países em desenvolvimento até o final da década de 1990 (Hackett \& Hackett, 1999). A partir do ano 2000, estudos epidemiológicos começaram a ser realizados em países em desenvolvimento, incluindo estudos no Brasil e outros países da América Latina, revelando taxas de prevalência de problemas de saúde mental (PSM) na infância e adolescência semelhantes aos países desenvolvidos (Belfer, 2008). Assim, estimativas atuais apontam que uma entre quatro a cinco crianças e adolescentes no mundo sofre algum transtorno mental (Patel, Flisher, Hetrick \& McGorry, 2007). No que tange o surgimento dos PSM ao longo do desenvolvimento humano, sabe-se que há influências de múltiplos e diversos precursores, compreen-

1 Apoio: Fundo de Auxílio aos Docentes e Alunos da UNIFESP e International Clinical Epidemiology Network. Apoio institucional: Universidade Presbiteriana Mackenzie e Universidade Federal de São Paulo (UNIFESP)

2 Endereço para correspondência: Rua da Consolação, 930. Edifício 38 Consolação, São Paulo, SP. CEP 01302-907. Fone (21) 2114-8707.

E-mail: csilvestrep@uol.com.br dendo aspectos biológicos, sociais e psicológicos diversos. Entre estes, a literatura científica destaca a importância que os fatores de risco exercem sob a saúde mental (Kazdin, Kraemer, Kessler, Kupfer \& Offord, 1997; Rutter, 1988).

Fatores de risco podem ser definidos como a presença de uma característica, experiência ou evento que aumenta a probabilidade da ocorrência de um dado desfecho quando comparado a mesma população não exposta. Em outras palavras, indivíduos expostos a um fator de risco podem apresentar vulnerabilidade para o surgimento de determinado desfecho clínico, constituindo um grupo vulnerável (Fletcher, Fletcher \& Wagner, 1996; Jekel, Katz \& Elmore, 2005; Kazdin \& cols., 1997).

A pesquisa de fatores de risco para PSM em crianças e adolescentes é fundamental para o campo da saúde pública. $\mathrm{O}$ conhecimento de potenciais fatores de risco e grupos vulneráveis traz a possibilidade de desenvolvimento de programas de intervenção focados em prevenir ou atenuar os efeitos deletérios destes na saúde mental de crianças e adolescentes (O'Connel, Boat \& Warner, 2009; OMS, 2001; OMS, 2005). Neste sentido, a epidemiologia oferece 
ferramentas para realizar investigações acerca de fatores de risco para PSM na infância e adolescência. A epidemiologia é uma disciplina focada no estudo do padrão de distribuição de doenças e fatores associados a estas em um determinado espaço e tempo. Esta linha teórica permite o levantamento de dados empíricos que podem ser usados como guias para intervenções, no sentido de prevenir e tratar doenças (Jekel \& cols., 2005; Medronho, 2006).

Visando compreender o cenário atual no que tange estudos epidemiológicos brasileiros, foi feito um levantamento bibliográfico sobre pesquisas nacionais que incluíram a investigação de fatores de risco para PSM em crianças e adolescentes. Segundo uma recente revisão da literatura científica, foram publicados nove estudos epidemiológicos populacionais na área da saúde mental da infância e adolescência no Brasil, no período de 1980 a 2006 (Bordin \& Paula, 2006). Essa revisão se restringiu a estudos populacionais que preencheram os seguintes critérios de inclusão: apresentar resultados baseados em amostra probabilística populacional com perda inferior a $40 \%$, referir estimativas de prevalência para PSM geral e utilizar instrumentos estruturados, desenvolvidos especificamente para avaliar a saúde mental de crianças e adolescentes. A seguir serão descritos todos os estudos identificados na citada revisão que trataram de fatores de risco para PSM na infância e adolescência.

Uma pesquisa realizada em três áreas distintas de Campos do Jordão-SP, (Fleitlich \& Goodman, 2001) verificou que pobreza, violência familiar e PSM maternos são fatores associados à PSM em crianças e adolescentes na faixa etária de sete a $14 \operatorname{anos}(\mathrm{N}=898)$. No total, 15\% dos escolares apresentaram PSM com base na escala de total de problemas do instrumento Strengths and Difficulties Questionnaire (SDQ) aplicado a pais e/ou professores. A taxa de PSM entre os moradores da favela $(22 \%)$ foi maior que as taxas observadas entre os sujeitos que habitavam as áreas urbanas $(12 \%)$ e rurais $(12 \%)$.

Posteriormente, um estudo de corte transversal foi conduzido em Taubaté-SP com uma amostra probabilística estratificada de estudantes de escolas públicas (urbanas e rurais) e particulares (todas urbanas), envolvendo 1251 crianças e adolescentes (entre sete e 14 anos) de todas as classes sociais (Fleitlich-Bilyk \& Goodman, 2004). Avaliando uma subamostra ( $\mathrm{N}=454$; faixa etária de sete a 11 anos) desse estudo, Vitolo, Fleitlich-Bilyk, Goodman e Bordin (2005) observaram que os PSM em geral, segundo a informação dos pais/cuidadores, estavam associados a quatro fatores de risco: (1) criança/adolescente ser do gênero masculino, (2) famílias onde pais/cuidadores afirmaram bater com o cinto em seus filhos, (3) presença de PSM nos pais/cuidadores e (4) pertencer a famílias com condições socioeconômicas desfavoráveis. Quando considerados tipos específicos de PSM, meninos apresentaram mais hiperatividade/déficit de atenção e problemas de relacionamento com colegas. Crianças que pertenciam a classes sociais mais desfavorecidas apresentaram mais problemas de conduta e problemas emocionais (ansiedade/depressão). Problemas de conduta foram mais freqüentes entre as crianças cujos pais batiam com cinto. Além disso, crianças que tinham pais/cuidadores com PSM apresentaram mais problemas de conduta, problemas emocionais e dificuldades de relacionamento com colegas. Por último, foi encontrada uma interação entre gênero e nível socioeconômico, revelando que nas classes D-E, os sintomas de ansiedade/depressão eram mais freqüentes entre as meninas, enquanto nas classes A-B-C, os problemas de relacionamento com colegas eram mais freqüentes entre os meninos.

Em Pelotas, Rio Grande do Sul, Benvegnú, Fassa, Facchini, Wegman e Dall'Agnol (2005), realizaram um estudo populacional objetivando investigar possíveis associações entre trabalho e PSM em crianças e adolescentes $(\mathrm{N}=3119)$ residentes em áreas de baixa renda. Os PSM (13,5\%) foram identificados com base na categoria clínica (escore $\mathrm{T}>63$ ) da escala de total de problemas do Child Behavior Checklist (CBCL). Começar a trabalhar muito cedo foi considerado importante fator associado a PSM. Entretanto, ainda que o trabalho doméstico estivessse associado a PSM entre crianças, no grupo dos adolescentes trabalhar foi um fator de proteção para PSM. Isto é, adolescentes que trabalhavam apresentavam menor taxa de PSM do que adolescentes que não trabalhavam. Outros fatores estiveram associados aos PSM, incluindo o consumo de álcool por parte de crianças e o hábito de fumar entre crianças/adolescentes, as reações maternas inadequadas (gritar, bater, punir severamente) frente ao mau comportamento dos filhos, a morbidade psiquiátrica materna e o baixo nível socioeconômico familiar.

Deste modo, apesar de escassas, pesquisas epidemiológicas brasileiras indicam que há associação entre fatores de riscos específicos e PSM na infância e adolescência. As evidências mais consistentes parecem ser em relação a crianças/ adolescentes em situação de vulnerabilidade como pobreza e vítimas de punição física. A literatura internacional aponta ainda que filhos de mulheres com depressão constituem grupo de risco para PSM, incluindo problemas emocionais e de comportamento (Ferro, Verdeli, Pierre \& Weissman, 2000; Klimes-Dougan \& cols., 1999; Pilowsky \& cols., 2006). Sabe-se também que mulheres vítimas de violência conjugal estão mais sujeitas a apresentar sintomas de ansiedade/ depressão e ideação suicida (Pico-Alfonso \& cols., 2006), portanto nos lares onde a violência conjugal está presente, as crianças devem estar mais sujeitas a desenvolver PSM.

Considerando a escassez de estudos epidemiológicos que investiguem fatores de risco para PSM na infância e adolescência no Brasil, a presente pesquisa visa identificar fatores de risco associados a estes problemas em uma comunidade urbana de baixa renda. Resultados deste e de outros estudos no tema poderão produzir evidências científicas com implicações pragmáticas promissoras. A exposição a fatores de risco torna crianças e adolescentes vulneráveis ao surgimento de PSM, portanto a identificação destes fatores pode contribuir para prevenir ou minimizar PSM (O'Connel \& cols., 2009; OMS, 2005).

\section{Método}

Este estudo de corte transversal constituiu a fase piloto do Estudo Brasileiro de Violência Doméstica contra a Criança e o Adolescente, vinculado à pesquisa multicêntrica internacional World Studies of Abuse in the Family Environment (WorldSAFE) (Bangdiwala, Paula, Ramiro \& Munoz, 2003). O presente estudo foi conduzido no Jardim Santa Emília, um típico bairro 
de baixa renda do município de Embu, região metropolitana de São Paulo. O projeto foi aprovado pelo Comitê de Ética em Pesquisa da Universidade Federal de São Paulo.

\section{Local da Coleta}

O município de Embu, com mais de 200.000 habitantes, é caracterizado pela baixa renda e baixa escolaridade da população, pela grande proporção de migrantes provenientes de regiões pobres do país, altos índices de violência urbana e presença do narcotráfico. Em 2002, enquanto o Estado de São Paulo apresentava taxa de 38,93 homicídios para cada 100.000 habitantes, o município de Embu registrava taxa quase três vezes superior (100,79 homicídios/100.000 habitantes) (SEADE, 2002).

\section{Participantes}

O estudo baseou-se em amostra probabilística de domicílios do município do Embu. O Instituto Brasileiro de Geografia e Estatística (IBGE) sorteou duas áreas geográficas (conglomerados) com base em unidades censitárias. Esses conglomerados são similares a maioria dos outros que compõem o município que é, de forma geral, homogêneo. Nesses dois conglomerados, foram identificados todos os domicílios elegíveis $(\mathrm{N}=100)$, ou seja, todos aqueles tendo como residentes pelo menos uma mãe de 15-49 anos com pelo menos um filho menor de 18 anos. Nos domicílios com mais de uma mãe e/ou mais de um filho nas faixas etárias pré-estabelecidas, ocorreram sorteios para a identificação de apenas uma mãe índice e uma criança índice. A amostra final do estudo piloto do BrazilSAFE incluiu 89 pares mãe-criança de zero a 17 anos de idade (perda amostral de $11 \%$ ). O presente estudo restringiu-se à subamostra de todas as crianças/ adolescentes de quatro a 17 anos de idade $(\mathrm{N}=67)$, faixa etária apropriada à aplicação do questionário de avaliação de PSM na infância e adolescência. $O$ pequeno tamanho amostral não permite afirmar que se trata de uma amostra estatisticamente representativa de todas as crianças e adolescentes da região. Por outro lado, devido ao cuidadoso processo de aleatorização empregado na seleção dos casos, é possível concluir que os resultados decorrentes deste estudo são aplicáveis a outras crianças/adolescentes moradoras da região, considerada homogênea em suas características sociodemográficas.

Após assinarem o Termo de Consentimento Livre e Esclarecido, as mães foram entrevistadas uma única vez individualmente no centro de saúde do bairro (com duração média de uma hora e meia), por entrevistadores treinados. Todos os casos suspeitos de violência intrafamiliar e/ou problemas de saúde mental foram devidamente encaminhados para os serviços públicos especializados da região.

\section{Instrumentos}

O Child Behavior Checklist (CBCL), instrumento na área da saúde mental da infância e adolescência mais utilizado internacionalmente (Duarte \& cols., 2003), foi aplicado a mães para identificar PSM nas crianças e adolescentes, segundo três desfechos clínicos: problemas do tipo internalização, problemas do tipo externalização e total de problemas em nível clínico (escore $\mathrm{T}>63$ ). Os problemas do tipo internalização são caracterizados por problemas internos ou emocionais, como ansiedade e depressão; enquanto os problemas do tipo externalização podem ser definidos como dificuldade em seguir regras sociais e/ou problemas do tipo disruptivos, como agressividade e violação de regras (Caputo \& Bordin, 2007). Nesse estudo, utilizou-se a versão brasileira do CBCL apropriadas para a faixa etária de quatro a 18 anos de idade (Achenbach, 1991). Dados preliminares de validação desta versão brasileira, segundo estudos conduzidos em São Paulo e no Rio de Janeiro, revelaram sensibilidade de $80,4 \%$, quando aplicado a mães de baixa escolaridade por entrevistador leigo treinado (Bordin, Mari \& Caeiro, 1995; Brasil, 2003).

A seguir serão descritos os instrumentos estruturados utilizados para identificar uma série de fatores potencialmente associados à PSM nas crianças/adolescentes.

O WorldSAFE Core Questionnaire (CORE) é um instrumento estruturado desenvolvido pelo comitê de pesquisadores de todos os países participantes do WorldSAFE (Sadowski L, Hunter W, Runyan D, Bangdwala K, Bordin I, Munhoz S, Hassan F, Jain D, Jeyaseelan L, Ramiro L). O Core Questionnaire foi traduzido e retro-traduzido para o português por Isabel Bordin e Cristiane S. de Paula em 1998 e engloba vários dados familiares e sociodemográficos, entre eles, punição física contra criança/adolescente e fatores sócio-demográficos como idade, escolaridade e situação empregatícia dos pais. A seção relativa à punição física contra criança/adolescente é parcialmente baseada no instrumento Parent-Child Conflict Tactics Scales (Straus, Hamby, Finkelhor, Moore \& Runyan, 1998) com permissão dos autores. Punição física grave da criança/adolescente foi definida pela presença de pelo menos uma das seguintes atitudes da mãe e/ou de seu marido/companheiro: chutar, esganar colocando as mãos ou alguma outra coisa em volta do pescoço, sufocar com as mãos ou travesseiro, queimar, espancar (bater repetidamente com objeto ou punho) e ameaçar com faca ou revólver. Violência conjugal física grave nos últimos 12 meses foi definida como chute, soco ou espancamento da mulher pelo marido/companheiro residente no domicílio. Essas definições representam o resultado de um consenso decorrente de reuniões dos pesquisadores do WorldSAFE, portanto, referem-se a comportamentos de violência considerados graves em diversas culturas.

A ideação suicida por parte da mãe foi definida por sua resposta positiva no item: "alguma vez você já pensou em se matar?”. O Self-Report Questionnaire (SRQ-20) é um instrumento estruturado com 20 itens para identificar PSM em adultos na comunidade, especialmente adequado a populações de países em desenvolvimento (Beusenberg \& Orley, 1994). O SRQ-20 foi desenvolvido para detectar casos prováveis de depressão, ansiedade e transtornos mentais com queixas somáticas. $\mathrm{O}$ instrumento foi validado e sua confiabilidade atestada no Brasil, mais especificamente na cidade de São Paulo, apresentando sensibilidade de $83 \%$ e especificidade de $80 \%$. Indivíduos com escore total acima de sete são considerados casos positivos para PSM (Mari \& Williams, 1986). 
Os fatores potencialmente associados à PSM em crianças e adolescentes, investigados no presente estudo, incluíram variáveis relacionadas à criança/adolescente (gênero, idade, punição física grave nos últimos 12 meses); à mãe (escolaridade, desemprego, violência conjugal física grave nos últimos 12 meses, PSM, ideação suicida); e ao pai/padrasto (desemprego ou ausência, embriaguez nos últimos 12 meses).

Com o auxílio do programa SPSS (versão 10.0) foi realizada a análise univariada, utilizando os testes de qui-quadrado e exato de Fisher. Foi calculada a odds ratio (OR) com intervalo de confiança de 95\% (IC 95\%). Os fatores considerados estatisticamente significantes foram aqueles com valor de $\mathrm{p}$ inferior a 0,05 .

\section{Resultados}

A amostra do presente estudo foi composta por 67 crianças e adolescentes com idade variando de quatro a 17 anos (média: 10,0 $\pm 3,9$ ), sendo 38 crianças de quatro a 10 anos (56,7\%), 29 adolescentes de 11 a 17 anos (43,3\%), 39 meninos $(58,2 \%)$ e 28 meninas $(41,8 \%)$. O nível socioeconômico das famílias era predominantemente baixo, segundo indicadores como escolaridade materna (44,8\% das mães não haviam completado a primeira série do ensino fundamental) e desemprego paterno (em 32,8\% dos domicílios o marido/ companheiro da mãe não trabalhava ou estava ausente).

Nessa amostra de crianças e adolescentes, identificamos as seguintes taxas de PSM segundo CBCL: 19,4\% de problemas do tipo internalização, 20,9\% de problemas do tipo externalização e 22,4\% de total de PSM. As características da amostra encontram-se resumidas na Tabela 1. Ressaltamos as altas taxas de violência intrafamiliar grave $(9,0 \%$ das crianças/adolescentes sofreram punição física grave e $9,0 \%$ das mães foram vítimas de violência conjugal física grave) e de PSM das mães segundo SRQ-20 (47,8\% das mães apresentavam sintomas sugestivos de ansiedade/depressão, $23,9 \%$ das mães já haviam pensado em suicídio). Em relação ao pai/padrasto, 32,8\% destes haviam ficado embriagados alguma vez nos últimos 12 meses.

A seguir descreveremos a distribuição dos fatores de risco estudados em relação aos desfechos clínicos na amostra do presente estudo. Considerando o gênero das crianças/ adolescentes entre os casos de crianças/adolescente com PSM, houve predominância de meninos com PSM do tipo internalização $(84,6 \%)$ e do tipo total de problemas $(73,3 \%)$. A faixa etária de quatro a 10 anos teve maior número de casos com PSM do tipo externalização (64,3\%) em relação à faixa etária de 11-17 anos. A maior prevalência de exposição à violência física grave contra criança/adolescente se deu entre casos com PSM do tipo externalização $(21,4 \%)$ e total de problemas $(26,7 \%)$. A violência conjugal física grave apresentou distribuição semelhante entre os casos de crianças/adolescentes com PSM (23,1\% do tipo internalização, $28,6 \%$ do tipo externalização e $26,7 \%$ do tipo total de problemas). Entre os casos de crianças/adolescentes com PSM que tinham mães que apresentavam PSM segundo SRQ-20, os PSM do tipo internalização $(61,5 \%)$ e total de problemas $(60 \%)$ foram os mais prevalentes. Por fim, considerando as crianças/adolescentes com PSM que tinham pai/ padrasto que se embriagou nos últimos 12 meses, o PSM do tipo internalização foi o que apresentou maior prevalência (61,5\%). A descrição pormenorizada de todos os fatores de risco investigados, segundo os três tipos de PSM de crianças/ adolescentes, encontra-se nas Tabelas 2 e 3.

A análise univariada identificou fatores de risco para os três desfechos clínicos de interesse do estudo (Tabela 4). A ideação suicida materna e a violência conjugal física grave colocaram as crianças/adolescentes em maior risco para problemas do tipo externalização e total de problemas $(\mathrm{p}<0,05)$. A punição física grave contra a criança/adolescente se mostrou associada ao total de problemas $(p=0,02)$, enquanto gênero masculino $(p=0,03)$ e embriaguez do pai/ padrasto $(p=0,02)$ foram fatores associados aos problemas do tipo internalização. A violência conjugal física grave esteve

Tabela 1. Descrição da amostra $(\mathrm{N}=67)$

\begin{tabular}{lll}
\hline $\mathrm{N}$ & $\%$ \\
\hline
\end{tabular}

Criança/adolescente

Sexo

Masculino

$39 \quad 58,2$

Feminino

$28 \quad 41,8$

Idade

4-10 anos

$37 \quad 55,2$

11-17 anos

$30 \quad 44,8$

Problemas de Saúde Mental

Total de problemas

$15 \quad 22,4$

Escala de externalização

$14 \quad 20,9$

Escala de internalização

$13 \quad 19,4$

Punição física grave

Sim

Não

69,0

Mãe

Escolaridade

$\geq$ uma série completa

38

56,7

Nenhuma série completa

$29 \quad 43,3$

Desemprego

$34 \quad 56,7$

Problemas de Saúde Mental

32

47,8

Ideação suicida

16

23,9

Violência física contra mulher

6

9,0

Pai/padrasto

Desemprego

45

67,2

Embriaguez (pelo menos uma vez nos últimos 12 meses) 
Tabela 2. Distribuição dos fatores de risco individuais da amostra de crianças e adolescentes segundo problemas de saúde mental de acordo com o instrumento estruturado CBCL $(\mathrm{N}=67)$.

\begin{tabular}{lccccccc}
\hline Fatores & & \multicolumn{2}{c}{ Internalização } & \multicolumn{2}{c}{ Externalização } & \multicolumn{2}{c}{ Total de problemas } \\
Criança/adolescente & & Sem PSM & PSM & Sem PSM & PSM & Sem PSM & PSM \\
& & \multicolumn{2}{c}{$\mathrm{N}(\%)$} & \multicolumn{2}{c}{$\mathrm{N}(\%)$} & $\mathrm{N}(\%)$ \\
\hline \multirow{2}{*}{ Gênero } & Masculino & $28(51,9 \%)$ & $11(84,6 \%)$ & $30(56,6 \%)$ & $9(64,3 \%)$ & $28(53,8 \%)$ & $11(73,3 \%)$ \\
& Feminino & $26(48,1 \%)$ & $2(15,4 \%)$ & $23(43,4 \%)$ & $5(35,7 \%)$ & $24(46,2 \%)$ & $4(26,7 \%)$ \\
Idade & $0-10$ anos & $30(55,6 \%)$ & $7(53,8 \%)$ & $28(52,8 \%)$ & $9(64,3 \%)$ & $31(59,6 \%)$ & $6(40 \%)$ \\
& $11-17$ anos & $24(44,4 \%)$ & $6(46,2 \%)$ & $25(47,2 \%)$ & $5(35,7 \%)$ & $21(40,4 \%)$ & $9(60 \%)$ \\
Punição física grave & Presença & $5(9,3 \%)$ & $1(7,7 \%)$ & $3(5,7 \%)$ & $3(21,4 \%)$ & $2(3,8 \%)$ & $4(26,7 \%)$ \\
& Ausência & $49(90,7 \%)$ & $12(92,3 \%)$ & $50(94,3 \%)$ & $11(78,6 \%)$ & $50(96,2 \%)$ & $11(73,3 \%)$ \\
\hline
\end{tabular}

Tabela 3. Distribuição dos fatores de risco familiares (mãe e pai/padrasto) da amostra de crianças e adolescentes segundo problemas de saúde mental de acordo com o instrumento estruturado CBCL $(\mathrm{N}=67)$.

\begin{tabular}{|c|c|c|c|c|c|c|c|}
\hline & & \multicolumn{2}{|c|}{ Internalização } & \multicolumn{2}{|c|}{ Externalização } & \multicolumn{2}{|c|}{ Total de problemas } \\
\hline & & Sem PSM & PSM & Sem PSM & PSM & Sem PSM & PSM \\
\hline \multicolumn{8}{|l|}{ Mãe } \\
\hline \multirow[t]{2}{*}{ Escolaridade } & Menos de 1 ano & $21(38,9 \%)$ & $8(61,5 \%)$ & $24(45,3 \%)$ & $5(35,7 \%)$ & $21(40,4 \%)$ & $8(53,3 \%)$ \\
\hline & 1 ano ou mais & $33(61,1 \%)$ & $5(38,5 \%)$ & $29(54,7 \%)$ & $9(64,3 \%)$ & $31(59,6 \%)$ & $7(46,7 \%)$ \\
\hline \multirow[t]{2}{*}{ Desemprego } & Sim & $26(48,1 \%)$ & $8(61,5 \%)$ & $24(45,3 \%)$ & $10(71,4 \%)$ & $24(46,2 \%)$ & $10(66,7 \%)$ \\
\hline & Não & $28(51,9 \%)$ & $5(38,5 \%)$ & $29(54,7 \%)$ & $4(28,6 \%)$ & $28(53,8 \%)$ & $5(33,3 \%)$ \\
\hline \multirow[t]{2}{*}{$\begin{array}{l}\text { Violência conjugal } \\
\text { física grave }\end{array}$} & Presença & $3(5,6 \%)$ & $3(23,1 \%)$ & $2(3,8 \%)$ & $4(28,6 \%)$ & $2(3,8 \%)$ & $4(26,7 \%)$ \\
\hline & Ausência & $51(94,4 \%)$ & $10(76,9 \%)$ & $51(96,2 \%)$ & $10(71,4 \%)$ & $50(96,2 \%)$ & $11(73,3 \%)$ \\
\hline \multirow[t]{2}{*}{$\begin{array}{l}\text { Problemas de saúde } \\
\text { mental }\end{array}$} & Presença & $24(44,4 \%)$ & $8(61,5 \%)$ & $25(47,2 \%)$ & $7(50 \%)$ & $23(44,2 \%)$ & $9(60 \%)$ \\
\hline & Ausência & $30(55,6 \%)$ & $5(38,5 \%)$ & $28(52,8 \%)$ & $7(50 \%)$ & $29(55,8 \%)$ & $6(40 \%)$ \\
\hline \multirow[t]{2}{*}{ Ideação suicida } & Presença & $11(20,4 \%)$ & $5(38,5 \%)$ & $9(17 \%)$ & $7(50 \%)$ & $9(17,3 \%)$ & $7(46,7 \%)$ \\
\hline & Ausência & $43(79,6 \%)$ & $8(61,5 \%)$ & $44(83 \%)$ & $7(50 \%)$ & $43(82,7 \%)$ & $8(53,3 \%)$ \\
\hline \multicolumn{8}{|l|}{ Pai/padrasto } \\
\hline \multirow[t]{2}{*}{$\begin{array}{l}\text { Desemprego ou } \\
\text { ausência* }\end{array}$} & Sim & $18(33,3 \%)$ & $4(30,8 \%)$ & $18(34 \%)$ & $4(28,6 \%)$ & $16(30,8 \%)$ & $6(40 \%)$ \\
\hline & Não & $36(66,7 \%)$ & $9(69,2 \%)$ & $35(66 \%)$ & $10(71,4 \%)$ & $36(69,2 \%)$ & $9(60 \%)$ \\
\hline \multirow[t]{2}{*}{ Embriaguez** } & Presença & $14(25,9 \%)$ & $8(61,5 \%)$ & $15(28,3 \%)$ & $7(50 \%)$ & $15(28,8 \%)$ & $7(46,7 \%)$ \\
\hline & Ausência & $40(74,1 \%)$ & $5(38,5 \%)$ & $38(71,7 \%)$ & $7(50 \%)$ & $37(71,2 \%)$ & $8(53,3 \%)$ \\
\hline
\end{tabular}

* Sem trabalho remunerado ou não residente no domicílio vs. com trabalho remunerado e residente no domicílio

** Últimos 12 meses 
Tabela 4. Resultados da análise univariada para identificar fatores de risco para problemas de saúde mental segundo CBCL em crianças/adolescentes: problemas do tipo internalização, problemas do tipo externalização e total de problemas $(\mathrm{N}=67)$.

\begin{tabular}{|c|c|c|c|c|c|c|c|c|c|}
\hline \multirow{2}{*}{ Fatores } & \multicolumn{3}{|c|}{ Internalização } & \multicolumn{3}{|c|}{ Externalização } & \multicolumn{3}{|c|}{ Total de problemas } \\
\hline & OR & IC $95 \%$ & $\mathbf{p}$ & OR & IC $95 \%$ & $\mathbf{p}$ & OR & IC $95 \%$ & $\mathbf{p}$ \\
\hline \multicolumn{10}{|l|}{ Criança/adolescente } \\
\hline Gênero (masculino vs. feminino) & 5,11 & $1,03-25,26$ & 0,03 & $-\cdots$ & --- & ns & --- & --- & ns \\
\hline Idade (4-10 anos) & --- & --- & ns & --- & --- & ns & --- & --- & ns \\
\hline Punição física grave* & --- & --- & ns & $-\cdots$ & --- & ns & 9,09 & $1,48-56,03$ & 0,02 \\
\hline \multicolumn{10}{|l|}{ Mãe } \\
\hline Escolaridade (0 vs. 1 ano ou mais) & --- & --- & ns & --- & --- & ns & --- & --- & ns \\
\hline Desemprego & --- & --- & ns & 3,02 & $0,84-10,86$ & 0,08 & --- & --- & ns \\
\hline Violência conjugal física grave* & 5,10 & $0,90-29,00$ & 0,08 & 10,20 & $1,64-63,44$ & 0,02 & 9,09 & $1,48-56,03$ & 0,02 \\
\hline Problemas de saúde mental & --- & $-\cdots$ & ns & $-\cdots$ & --- & ns & --- & --- & ns \\
\hline Ideação suicida & --- & --- & ns & 4,89 & $1,37-17,40$ & 0,03 & 4,18 & $1,21-14,49$ & 0,04 \\
\hline \multicolumn{10}{|l|}{ Pai/padrasto } \\
\hline Desemprego ou ausência* & --- & --- & ns & --- & --- & ns & --- & --- & ns \\
\hline Embriaguez** & 4,57 & $1,28-16,32$ & 0,02 & $-\cdots$ & --- & ns & $-\cdots$ & --- & ns \\
\hline \multicolumn{10}{|c|}{ * Sem trabalho remunerado ou não residente no domicílio vs. com trabalho remunerado e residente no domicílio } \\
\hline \multicolumn{10}{|l|}{$\mathrm{ns}=$ não significativo $(\mathrm{p}>0,05)$} \\
\hline $\mathrm{OR}=$ Odds Ratio & & & & & & & & & \\
\hline
\end{tabular}

associada com total de problemas $(\mathrm{p}=0,02)$ e problemas do tipo externalização $(\mathrm{p}=0,02)$, além de apresentar tendência à associação com problemas do tipo internalização $(p=0,08)$. Finalmente, pode-se observar uma tendência à associação entre desemprego materno e problemas do tipo externalização $(\mathrm{p}=0,08)$.

Além disso, as mães que referiram ideação suicida tiveram probabilidade oito vezes maior de ter sofrido violência conjugal física grave nos últimos 12 meses em comparação com as mães que não referiram essas idéias $(p=0,03)$. As mães com ideação suicida também tiveram probabilidade quatro vezes maior de ter um marido/companheiro que se embriagou nos últimos 12 meses em comparação com as mães que não referiram embriaguez do marido/companheiro nos últimos 12 meses $(\mathrm{p}=0,02)$. Observou-se ainda que todas as mães que sofreram violência conjugal física grave nos últimos 12 meses referiram embriaguez do marido/companheiro no mesmo período.

\section{Discussão}

O presente estudo identificou a ideação suicida materna como fator de risco para PSM dos tipos externalização e total de problemas de crianças/adolescentes segundo CBCL. Após revisão, não identificamos na literatura estudos que tenham encontrado esta mesma associação. Por outro lado, não se identificou associação entre PSM maternos e PSM de crianças/adolescentes desta amostra, provavelmente devido à alta taxa de PSM maternos aqui presentes (47,8\%). Assim, a ideação suicida (com freqüência de $23,9 \%$ ) parece ter sido um marcador de gravidade da psicopatologia materna nesta amostra, mais sensível que os PSM maternos como um todo.

Considerando esta hipótese é possível fazer comparações entre os dados do presente estudo com outras pesquisas que investigaram PSM maternos como fator de risco para PSM dos filhos. Estudos brasileiros conduzidos com amostras de crianças/adolescentes de todas as camadas sociais relatam que a psicopatologia materna é um importante fator de risco para PSM nos filhos (Fleitlich \& Goodman, 2001; Goodman, Fleitlich-Bilyk, Patel \& Goodman, 2007; Vitolo \& cols., 2005).

No mais recente destes estudos (Goodman \& cols., 2007), casos clínicos foram identificados pelo instrumento SDQ, tendo como resultado a identificação de PSM nos pais como fator de risco para problemas emocionais (equivalentes aos problemas do tipo internalização) e problemas de conduta (equivalentes aos problemas do tipo externalização) de seus 
filhos. A literatura especializada indica que mães deprimidas provêm menos estímulos positivos, respondem menos a estímulos dos filhos e oferecem menos afeto aos filhos do que mães não deprimidas, por sua vez, seus filhos também respondem menos a estímulos, são menos atentos e ativos do que filhos de mães não deprimidas. Dessa forma, o cuidado materno pode ser prejudicado, aumentando a probabilidade de a criança desenvolver PSM, principalmente quando a depressão materna coincide com os primeiros anos de vida da criança (Field \& cols., 1985; Maughan, Cicchetti, Toth $\&$ Rogosch, 2007).

Chama atenção o fato de que todas as mães de nossa amostra que haviam sofrido violência conjugal física grave nos últimos 12 meses referiram embriaguez do marido/ companheiro no mesmo período. A relação entre uso de álcool, não exclusivamente abusivo, e violência conjugal física contra mulher também é reconhecida na literatura (Moreno Martín, 1999). Assim, este estudo indica que nas famílias onde existe um fator de risco para PSM de crianças e adolescentes, em geral, esse vem acompanhado de outros fatores de risco, constituindo um complexo cenário em que as crianças/adolescentes estão expostas a diversos fatores estressores. Este dado é importante, pois segundo Halpern e Figueiras (2004) o efeito cumulativo de múltiplos fatores é mais importante que a presença de fatores isolados, do mesmo modo que adversidades duradouras ou que se repetem com freqüência são as mais prejudiciais ao desenvolvimento das crianças.

A violência conjugal física grave sofrida pela mãe é um conhecido fator de risco para PSM da criança/adolescente (Ross, 1996). Em uma revisão de fatores de risco associados à PSM de crianças/adolescentes, Maia e Williams (2005) encontraram diversos estudos que apontam nessa direção, citando alguns dos efeitos nocivos para crianças/adolescentes que presenciam violência física entre seus pais, como: uso de álcool/drogas, distúrbios da atenção, baixo rendimento escolar, ansiedade, depressão, transtorno de estresse pós-traumático e problemas somáticos. Nota-se que essa gama de problemas perpassa os problemas do tipo internalização e externalização. Um estudo realizado por McFarlane, Groff, O'Brien e Watson (2003) revelou associação entre violência conjugal contra mulher e PSM dos filhos (internalização, externalização e total de problemas, segundo CBCL). Em nossa amostra foi encontrada associação com os três desfechos clínicos investigados, o que corrobora com outros achados da literatura internacional.

Vale ressaltar que numa família onde a violência conjugal está presente há maior probabilidade de que também esteja presente a violência física contra criança perpetrada por pais (Herrenkohl, Sousa, Tajima, Herrenkohl \& Moylan, 2008). Deste modo, o ambiente como um todo costuma ser violento, sendo, portanto, um local de risco para o desenvolvimento de PSM na infância e adolescência. A relação familiar serve como modelo para o aprendizado de padrões comportamentais e sociais das crianças, dessa forma, a violência conjugal atua como um modelo negativo, gerando comportamentos prejudiciais à interação social e, por sua vez, prejudicial à saúde mental das crianças e adolescentes que convivem com estes modelos (Bandura, 1976).
A presença de punição física grave contra crianças e adolescentes também se mostrou associada à PSM na amostra estudada, concordando com o que já havia sido apontado por outros estudos epidemiológicos internacionais (Kaplan \& cols., 1998; Shaw \& Krause, 2002; Springer, Sheridan, Kuo $\&$ Carnes, 2007) e nacionais (Benvegnú \& cols., 2005; Bordin \& Paula, 2006; Fleitlich \& Goodman, 2001; Vitolo \& cols., 2005). A violência física é, portanto, um grave e importante fator de risco para problemas de saúde mental na infância e adolescência (Kaplan \& cols., 1998; Krug \& cols., 2002; Cicchetti \& Toth, 2005; Springer, Sheridan, Kuo \& Carnes, 2007). Os efeitos da violência física contra crianças e adolescentes são amplamente discutidos na literatura científica e vão além dos PSM e dos danos físicos imediatos, mostrando indícios de que até mesmo problemas físicos na idade adulta podem ter relação com eventos de violência na infância e/ou adolescência (Springer \& cols., 2007).

Uma possível explicação para a relação entre a violência física contra a criança/adolescente e PSM se refere a alterações na regulação do afeto, conceito psicológico que implica o controle e modulação das emoções (Cicchetti \& Toth, 2005). Assim, respostas de medo e agressividade excessivas ou inadequadas por parte da criança e/ou adolescente frente à exposição à violência física, causariam uma trajetória de adaptação a estímulos afetivos e emocionais prejudicada. Isto possivelmente ocorre em razão de problemas em processos atencionais de crianças vítimas de violência, sendo essas mais sensíveis a estímulos agressivos. Dessa forma, tais problemas também afetariam o processamento de informações sociais e, por conseguinte, crianças e/ou adolescentes que sofreram violência física teriam a tendência a interpretar estímulos sociais não violentos com hostilidade. Em suma, problemas na regulação do afeto advindos da exposição à violência parecem contribuir para falhas no desenvolvimento da maturação psicológica da criança e do adolescente (Cicchetti \& Toth, 2005).

Além disso, há indícios científicos de que pais agressores possuem características comportamentais como dificuldade de manejo de raiva, ausência de empatia, menos interação com o filho e menor compreensão do papel parental (Maia \& Williams, 2005). Essas características prejudicam o cuidado dos pais em relação aos filhos, agravando os problemas causados pela violência física e criando um ambiente prejudicial ao desenvolvimento infantil.

O gênero masculino esteve associado a problemas do tipo internalização na presente amostra, contrariando achados da literatura (Goodman \& cols., 2007), já que o gênero feminino é o mais freqüentemente associado a problemas emocionais, principalmente na adolescência. Este resultado inesperado, provavelmente decorre das características de nossa amostra, com predomínio de crianças (quatro a 10 anos) e de meninos, refletindo uma das limitações deste estudo que é o pequeno tamanho amostral. Para melhor compreensão desse resultado, faz-se necessário a condução de novos estudos epidemiológicos nacionais na área da infầncia e adolescência com amostras maiores, com ampla faixa etária e com uma distribuição de gênero mais equilibrada que a do presente estudo.

Para finalizar, algumas limitações devem ser apontadas. O pequeno tamanho da amostra, já mencionado anteriormente, reduziu o poder estatístico da análise e por isso, alguns 
dados devem ser interpretados com cautela. Optamos por fazer uma análise univariada também em razão do tamanho da amostra, já que uma análise multivariada poderia produzir dados menos confiáveis. Em relação ao desenho de estudo, destacamos que estudos transversais não permitem estabelecer uma seqüência temporal de causa e efeito entre as variáveis e os desfechos investigados.

Este estudo identificou diversos fatores psicossociais associados à PSM na infância e adolescência que devem ser considerados em propostas de programas de promoção, prevenção e tratamento. O desenvolvimento de programas que promovam a saúde mental e atitudes parentais não violentas, somados ao tratamento de PSM na família, são fundamentais. Do mesmo modo que a integração dos cuidados a saúde mental ao sistema primário de saúde parece ser uma importante estratégia para a detecção precoce de PSM e de violência doméstica contra crianças, adolescentes e suas mães (OMS, 2001). Visitas domiciliares por parte de profissionais do sistema de saúde podem aumentar a sensação de apoio comunitário e de suporte emocional, além de colaborar para a identificação de problemas no campo da violência e da saúde mental. Todo esse cenário acaba por ampliar o alcance dos serviços de saúde e facilitar encaminhamentos adequados (Krug \& cols., 2002; OMS, 2001).

Como tratamento aos PSM na infância e/ou adolescência, a Organização Mundial de Saúde recomenda uma combinação de três abordagens, levando-se em conta a especificidade de cada caso: psicoterapia, farmacoterapia e reabilitação psicossocial. Por isso, um tratamento adequado leva em consideração uma estratégia traçada por uma equipe de saúde mental, posterior a um cuidadoso diagnóstico. Abordagens multidisciplinares que levem em conta características psicossociais da criança e/ou adolescente, sua família e comunidade também contribuem para um melhor prognóstico (OMS, 2001).

Do ponto de vista da prevenção, programas e campanhas sociais são as estratégias mais recomendadas. Por exemplo, programas voltados a divulgação do conhecimento à população em relação à PSM e ao desenvolvimento infantil podem surtir efeito a longo prazo. Programas preventivos focados na relação mãe-filho podem gerar resultados a curto e longo prazo, pois PSM maternos interferem no desenvolvimento da criança e/ou adolescente, conforme já discutido.

No que se refere à prevenção da violência física contra crianças e adolescentes, uma revisão sistemática de programas de prevenção de maus-tratos na infância/adolescência demonstrou que o programa de visitas domiciliares por profissionais formados em saúde mental (caracterizado por visitas a famílias de alto risco, visando identificar problemas e orientar os pais) é o de maior eficácia e, portanto, o mais recomendável (Fergusson, Grant, Horwood \& Ridder, 2005; Macmillan \& cols., 2009). Assim, profissionais de saúde bem treinados podem contribuir estimulando uma boa relação mãe-filho, ensinando práticas educativas alternativas à punição física e conscientizando quanto ao desenvolvimento da criança. Somadas a essas propostas, mudanças culturais e sociais, além do fortalecimento das áreas jurídicas e judiciárias, também podem contribuir para a prevenção da violência física (Krug \& cols., 2002; OMS, 2001).
Para finalizar, ressaltamos que a área da infância e adolescência tem complexidades específicas que precisam ser consideradas em propostas de políticas de saúde pública. Por exemplo, grande parte do atendimento à saúde mental infantil não ocorre exclusivamente no setor da saúde, mas envolve também os setores da educação, justiça e/ou cidadania e assistência social (Duarte \& cols., 2003). Além disso, os fatores de risco que contribuem para o desenvolvimento e o agravamento dos PSM na infância e adolescência são inúmeros, complexos e inter-relacionados (Halpern \& Figueiras, 2004). Portanto, conhecer uma determinada realidade é de grande importância, pois permite a detecção precoce de grupos vulneráveis e se este processo estiver articulado com propostas de tratamento, resultados promissores de reabilitação podem ser alcançados, já que o tratamento precoce tende a ser mais eficaz, evitando prejuízos ao desenvolvimento das crianças/ adolescentes e a piora do quadro clínico (Burns, Hoagwood \& Mrazek, 1999; O’Connel \& cols., 2009; OMS, 2001).

\section{Referências}

Achenbach, T. M. (1991). Integrative guide for the 1991 CBCL/4-18, $Y S R$, and TRF profiles. Manuscrito não publicado, Department of Psychiatry, University of Vermont, Vermont.

Bandura, A. (1976). Social learning theory. New Jersey: Prentice Hall.

Bangdiwala, S. I.; Paula, C. S.; Ramiro, L. S., \& Munoz, S. R. (2003). Coordination of international multicenter studies: governance and administrative structure. Salud Pública de México, 45, 58-66.

Belfer, M. L. (2008). Child and adolescent mental disorders: the magnitude of the problem across the globe. Journal of Child Psychology and Psychiatry, 49, 226-236.

Benvegnú, L. A.; Fassa, A. G.; Facchini, L. A.; Wegman, D. H., \& Dall'Agnol, M. M. (2005). Work and behavioural problems in children and adolescents. International Journal of Epidemiology, $34,1417-1424$.

Beusenberg, M., \& Orley, J. H. (1994). A User's guide to the self reporting questionnaire (SRQ). Geneva: Organização Mundial da Saúde.

Bordin, I. \& Paula, C. (2006). Estudos Populacionais sobre Saúde Mental de Crianças e Adolescentes Brasileiros. Em M.F. Mello, A.A. Mello \& R. Kohn (Orgs.), Epidemiologia da Saúde Mental no Brasil (pp. 119-142). Porto Alegre: Artmed.

Bordin, I. A., Mari, J. J., \& Caeiro, M. F. (1995). Validação da Versão Brasileira do Child Behavior Checklist (CBCL) (Inventário de Comportamentos da Infância e Adolescência): Dados Preliminares. Revista Brasileira de Psiquiatria, 17, 55-66.

Brasil, H. H. A. (2003). Desenvolvimento da versão brasileira da K-SADS-PL (Schedule for Affective Disorders and Schizophrenia for School Aged Children: Present and Lifetime Version) e estudo de suas propriedades psicométricas. Tese de Doutorado, Universidade Federal de São Paulo, São Paulo.

Burns, B.; Hoagwood, K., \& Mrazek, P. (1999). Effective treatment for mental disorders in children and adolescents. Clinical Child and Family Psychology Review, 2, 199-254.

Caputo, V., \& Bordin, I. (2007). Mental health problems among pregnant and non-pregnant youth. Revista de Saúde Pública, $41,573-581$. 
Cicchetti, D., \& Toth, S. L. (2005). Child maltreatment. Annual Review of Clinical Psychology, 1, 409-438.

Duarte, C.; Hoven, C.; Berganza, C.; Bordin, I.; Bird, H., \& Miranda, C. T. (2003). Child mental health in Latin America: present and future epidemiologic research. International Journal of Psychiatry in Medicine, 33, 203-222.

Fergusson, D. M.; Grant, H.; Horwood, L. J., \& Ridder, E. M. (2005). Randomized trial of the Early Start program of home visitation. Pediatrics, 116, 803-809.

Ferro, T.; Verdeli, H.; Pierre, F., \& Weissman, M. M. (2000). Screening for depression in mothers bringing their offspring for evaluation or treatment of depression. American Journal of Psychiatry, 157, 375-379.

Field, T., Sandberg, D., Garcia, R., Vega-Lahr, N., Goldstein, S., \& Guy, L. (1985). Pregnancy problems, postpartum depression and early mother-infant interactions. Developmental psychology, 21, 1152-1156.

Fleitlich-Bilyk, B., \& Goodman, R. (2004). Prevalence of child and adolescent psychiatric disorders in southeast Brazil. Journal of the American Academy of Child and Adolescent Psychiatry, 43, 727-734.

Fleitlich, B., \& Goodman, R. (2001). Social factors associated with child mental health problems in Brazil: cross sectional survey. British Medical Journal, 323, 599-600.

Fletcher, R. H., Fletcher, S. W., \& Wagner, E. H. (1996). Epidemiologia clínica: elementos essenciais ( $3^{\circ}$ ed.). Porto Alegre: Artes Médicas.

Goodman, A., Fleitlich-Bilyk, B., Patel, V., \& Goodman, R. (2007). Child, family, school and community risk factors for poor mental health in Brazilian schoolchildren. Journal of the American Academy of Child and Adolescent Psychiatry, 46, 448-456.

Hackett, R., \& Hackett, L. (1999). Child Psychiatry across cultures. International Review of Psychiatry, 11, 225-235.

Halpern, R., \& Figueiras, A. C. (2004). Environmental influences on child mental health. Jornal de Pediatria, 80, S104-110.

Herrenkohl, T. I., Sousa, C., Tajima, E. A., Herrenkohl, R. C., \& Moylan, C. A. (2008). Intersection of child abuse and children's exposure to domestic violence. Trauma, Violence and Abuse, 9, 84-99.

Jekel, J. F., Katz, D. L., \& Elmore, J. G. (2005). Epidemiologia, bioestatística e medicina preventiva ( $2^{\circ}$ ed.). Porto Alegre: ArtMed.

Kaplan, S. J., Pelcovitz, D., Salzinger, S., Weiner, M., Mandel, F. S., Lesser, M. L., \& Labruna, B.A. (1998). Adolescent physical abuse: risk for adolescent psychiatric disorders. American Journal of Psychiatry, 155, 954-959.

Kazdin, A. E.; Kraemer, H. C.; Kessler, R. C.; Kupfer, D. J., \& Offord, D. R. (1997). Contributions of risk-factor research to developmental psychopathology. Clinical Psychology Review, 17, 375-406.

Klimes-Dougan, B.; Free, K.; Ronsaville, D.; Stilwell, J.; Welsh, C. J., \& Radke-Yarrow, M. (1999). Suicidal ideation and attempts: a longitudinal investigation of children of depressed and well mothers. Journal of the American Academy of Child and Adolescent Psychiatry, 38, 651-659.

Krug, E. G.; Dahlberg, L. L.; Mercy, J. A.; Zwi, A. B.; Lozano, R., \& World Health Organization (Orgs.). (2002). World report on violence and health. Geneva: World Health Organization.
Macmillan, H. L., Wathen, C. N., Barlow, J., Fergusson, D. M., Leventhal, J. M., \& Taussig, H. N. (2009). Interventions to prevent child maltreatment and associated impairment. Lancet, $373,250-266$.

Maia, J., \& Williams, L. (2005). Fatores de risco e fatores de proteção ao desenvolvimento infantil: uma revisão da área. Temas em psicologia, 13, 91-103.

Mari, J.J., \& Williams, P. (1986). A validity study of a psychiatric screening questionnaire (SRQ-20) in primary care in the city of Sao Paulo. British Journal of Psychiatry, 148, 6.

Maughan, A., Cicchetti, D., Toth, S. L., \& Rogosch, F. A. (2007). Early-occurring maternal depression and maternal negativity in predicting young children's emotion regulation and socioemotional difficulties. Journal of Abnormal Child Psychology, 35, 685-703.

McFarlane, J. M., Groff, J. Y., O’Brien, J. A., \& Watson, K. (2003). Behaviors of children who are exposed and not exposed to intimate partner violence: an analysis of 330 black, white, and Hispanic children. Pediatrics, 112, 202-207.

Medronho, R. A. (2006). Epidemiologia (1 ${ }^{\circ}$ ed.). Rio de Janeiro: Atheneu.

Moreno Martín, F. (1999). La violencia en la pareja. Revista panamericana de salud pública, 5, 245-258.

O’Connel., M. E., Boat., T., \& Warner., K. E. (Orgs.) (2009). Preventing mental, emotional, and behavioral disorders among young people: progress and possibilities. Washington, DC: National Academies Press.

Organização Mundial da Saúde. (2001). The World health report 2001: Mental health: new understanding, new hope. Geneva: World Health Organization.

Organização Mundial da Saúde, Departamento de Saúde Mental e Uso de Substancias (2005). Promoting mental health : concepts, emerging evidence, practice: a report of the World Health Organization. Genebra: Autor.

Patel, V., Flisher, A. J., Hetrick, S., \& McGorry, P. (2007). Mental health of young people: a global public-health challenge. Lancet, 369, 1302-1313.

Pico-Alfonso, M. A., Garcia-Linares, M. I., Celda-Navarro, N., Blasco-Ros, C., Echeburua, E., \& Martinez, M. (2006). The impact of physical, psychological, and sexual intimate male partner violence on women's mental health: depressive symptoms, posttraumatic stress disorder, state anxiety, and suicide. Journal of Women's Health (Larchmt), 15, 599-611.

Pilowsky, D. J., Wickramaratne, P. J., Rush, A. J., Hughes, C. W., Garber, J., Malloy, E., King, C., Cerda, G., Bela Sood, A., Alpert, J.E., Wisniewski, S., Trivedi, M.H., Talati, A., Carlson, M., Liu, H.H., Fava, M., \& Weissman, M.M. (2006). Children of currently depressed mothers: a STAR*D ancillary study. Journal of Clinical Psychiatry, 67(1), 126-136.

Ross, S. M. (1996). Risk of physical abuse to children of spouse abusing parents. Child Abuse and Neglect, 20, 589-598.

Rutter, M. (1988). Studies of psychosocial risk - the power of longitudinal data. Cambridge: Cambridge University Press.

Sistema Estadual de Análise de Dados (2002). Informações dos municipios paulistas. Retirado de http://www.seade.gov.br

Shaw, B. A., \& Krause, N. (2002). Exposure to physical violence during childhood, aging, and health. Journal of Aging and Health, 14, 467-494. 
Springer, K. W., Sheridan, J., Kuo, D., \& Carnes, M. (2007). Longterm physical and mental health consequences of childhood physical abuse: results from a large population-based sample of men and women. Child Abuse and Neglect, 31, 517-530.

Straus, M. A., Hamby, S. L., Finkelhor, D., Moore, D. W., \& Runyan, D. (1998). Identification of child maltreatment with the ParentChild Conflict Tactics Scales: development and psychometric data for a national sample of American parents. Child Abuse and Neglect, 22, 249-270.
Vitolo, Y. L., Fleitlich-Bilyk, B., Goodman, R., \& Bordin, I. A. (2005). Parental beliefs and child-rearing attitudes and mental health problems among schoolchildren. Revista de Saúde Pública, 39, 716-724.

Recebido em 03.11 .08

Primeira decisão editorial em 29.06.10

Versão final em 30.07.10

Aceito em 14.10.10 